\title{
GRAFICACIÓN DE ECUACIONES LINEALES CONTINUAS EN 2D USANDO EL MÉTODO DE CÁLCULO DE PENDIENTES
}

\section{DRAWING OF CONTINUOUS 2D LINEAL EQUATIONS USING THE PENDING CALCULATION METHOD}

JORGE ALARCÓN MENA ${ }^{1}$

Recibido: 15 de septiembre de 2017 Aceptado: 31 de enero de 2018

\footnotetext{
${ }^{1}$ Pontificia Universidad Católica del Ecuador, Facultad de Ingeniería, Quito, Ecuador (jalarcon@puce.edu.ec).
} 
illl| 


\section{GRAFICACIÓN DE ECUACIONES LINEALES CONTINUAS EN 2D USANDO EL MÉTODO DE CÁLCULO DE PENDIENTES}

\section{DRAWING OF CONTINUOUS 2D LINEAL EQUATIONS USING THE PENDING CALCULATION METHOD}

Jorge Alarcón Mena

Palabras Clave: Graficación de ecuaciones, ecuaciones lineales, pendientes, pixeles, PictureBox, transformación de puntos.

Keywords: Equation drawing, lineal equations, pending, pixels, PictureBox, point transformation.

\section{RESUMEN}

El presente documento propone un método para dibujar ecuaciones lineales continuas sobre un objeto gráfico visual en un lenguaje de programación, a través del uso de transformaciones de coordenadas basadas en el método físico de transformación de escalas termométricas. 


\section{ABSTRACT:}

This document proposes how to draw continues lineal equations on a graphical visual object using a pro- gramming language, using coordinates transformations upon on thermometric physics scales.

\section{INTRODUCCIÓN}

Uno de los temas que normalmente no se trata cuando se está estudiando programación visual, pero que la ingeniería en general lo considera muy importante es la graficación de ecuaciones lineales. Esto se puede deber a que el tema mencionado es muy específico y aparentemente de no mucha importancia para los cursos iniciales de programación, además; a ello se suma el hecho que en la actualidad existe una infinidad de paquetes que pueden hacer dicha tarea, no solo en 2, sino en 3 dimensiones.

Es común que mientras se estudian temas relacionados con programación orientada a objetos y programación visual, lo importante es prestar atención a los principios que fundamentan la programación orientada a objetos y al modo de trabajar con los diferentes objetos visuales en lo que respecta a la programación visual. Adicionalmente, la gran cantidad de objetos que existen en la actualidad y que, a groso modo, son necesarios de aprender a utilizar; suelen evitar que se profundice adecuadamente con las posibilidades de varios de ellos.

En este contexto, el presente apartado pretende dar una utilización específica y práctica de lo que se podría considerar un aspecto bastante específico del uso de objetos gráficos para la graficación de ecuaciones lineales en dos dimensiones mediante una técnica basada en la física tradicional, con el fin de tener una alternativa académica que pueda ayudar al estudiante a comprender cómo principios fundamentales de una rama de la ciencia pueden ser usados como base para solucionar problemas informáticos específicos. 


\section{GRAFICACIÓN DE ECUACIONES LINEALES CONTINUAS EN 2D USANDO EL MÉTODO DE CÁLCULO DE PEN- DIENTES}

Existen varios modos de dibujar ecuaciones en objetos gráficos mediante pixeles (Rex B. Kline, 2015, Principles and Practice of Structural Equation Modeling), por lo que este apartado constituye una propuesta con un enfoque diferente, utilizando principios de Física.

La idea básica es transformar cada uno de los puntos de la ecuación en puntos correspondientes dentro de los límites de un objeto gráfico. El proceso de transformación es similar al proceso que se usa en física para transformar escalas termométricas (Núñez Miguel, 2002, Física 2), pero aplicado a la informática bajo los mismos criterios. Para ello hay algunas condiciones iniciales:

- Debe tratarse de una ecuación lineal, independiente del grado $u$ orden.

- La sección de la ecuación que se pretenda graficar debe ser continua, es decir no deben existir rangos para los cuales la ecuación carezca de solución, porque aplicar el mecanismo para estos rangos llevaría a desbordamientos en los cálculos.

- Se deben determinar los límites máximos y mínimos en el plano cartesiano, para los cuales se pretende dibujar la ecuación. Para facilitar la explicación del proceso, a partir de este momento a la representación por pantalla del plano cartesiano simplemente se la llamará la pantaIla (Fernández Horacio, 2005, Matemáticas Previas al Cálculo).

- Se deben tener claros los puntos límites del objeto gráfico informático (comúnmente un PictureBox) sobre el cuál se va a graficar la ecuación (Navarro Rocío, 2012, Sistemas de Ecuaciones).

- Cada cuadrante de la ecuación se analiza, procesa y dibuja por separado.

- Se debe considerar que la coordenada $(0,0)$ de la ecuación no necesariamente será la coordenada $(0,0)$ en el objeto de graficación.

\section{Procedimiento:}

El mecanismo que se detalla a continuación es aplicable a cualquier ecuación que cumpla con los criterios ya descritos, pero para efectos prácticos, en aquellos puntos en los que se requiera un ejemplo concreto se lo hará usando la siguiente ecuación:

$$
\frac{1}{4} x^{3}+\frac{3}{4} x^{2}-\frac{3}{2} x-2=0
$$

Ecuación 1: Ecuación de ejemplo para aplicar el método 
Se ha tomado esta ecuación en particular porque aparte de cumplir con las condiciones anteriores, sus raíces son fáciles de calcular y son $\times 1=-4, \times 2=-1$, $\times 3=2$; además, la gráfica de la ecuación es apreciable en los 4 cuadrantes del plano cartesiano.

El procedimiento para el proceso propuesto es el siguiente:

1. Se definen los límites que tendrá cada cuadrante dentro del plano cartesiano para los ejes $x$ e y. Para el ejemplo que se está desarrollando y tomando en cuenta que las raíces nos ayudan a conocer cuáles deben ser los límites que se destinarán como límites del plano cartesiano los siguientes en $x$ desde -5 hasta 3, en y desde -4 hasta 4 . Los límites pueden ser los que se deseen, pero siempre es importante tomar límites que permitan apreciar claramente la ecuación. Para no confundir estos límites con los de la pantalla se los llamará Xmin, Xmax, Ymin, Ymax, y en ejemplo el ejemplo propuesto los valores serían: $X \min =-5, X \max =3$, $Y \min =-4, Y \max =4$.

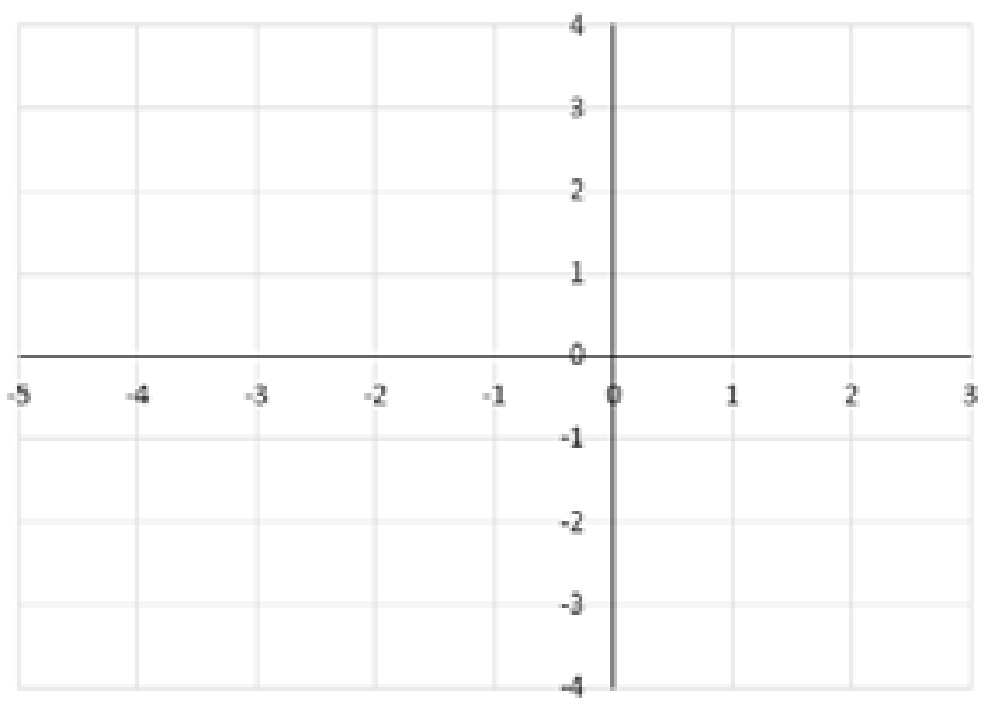

Figura 1. Plano cartesiano designado para la ecuación 
2. Se verifica que todo el plano elegido en el punto 1 entrará en el objeto gráfico (pantalla) en el cual se va a graficar la ecuación propuesta, la pantalla normalmente tiene como coordenada de origen $(0,0)$ la esquina superior izquierda y como coordenada máxima de la pantalla la esquina inferior derecha (Xpmax, Ypmax). En un objeto gráfico de di- bujo los puntos Xpmax y Ypmax son conocidos y son el ancho y el alto del objeto, respectivamente (Brice-Arnaudm, 2013, ASP.NET 4.5 en C\# Con Visual Studio 2012). Para no confundir estos puntos con los de la ecuación, siempre que se haga referencia a puntos dentro de la pantalla se colocará una p simbolizando la palabra pantalla.

$(0,0)$

Objeto en el cual se graficará la ecuación pixel a pixel

(Pantalla).

\section{(Xpmax, Ypmax)}

Figura 2. Límites de un objeto gráfico

En la Figura 1 se puede apreciar que el problema consiste en colocar puntos que la ecuación va a generar mediante el paso 1 en puntos correspon- dientes dentro de la pantalla (en realidad el objeto de dibujo), considerando los límites del plano cartesiano y de la pantalla, que no son los mismos. Esto es más 
visible si superponemos las figuras 2 y 3 , coincidan, tal como se lo puede apreciar una sobre la otra para que sus contornos en la Figura 3.

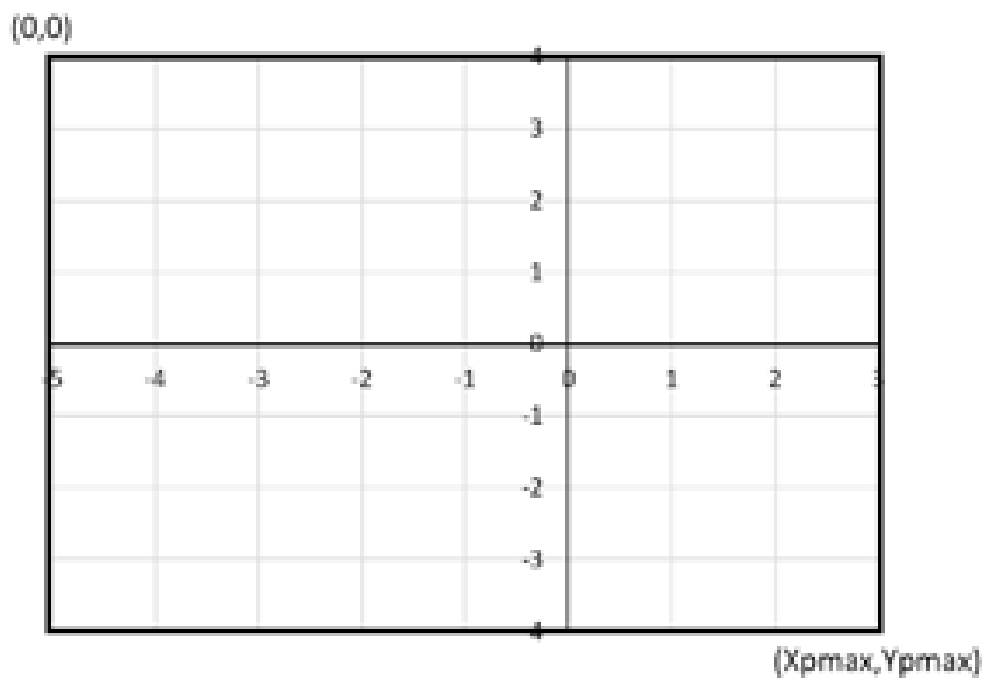

Figura 3. Superposición de las Figuras 1 y 2

3. Se colocan etiquetas en los puntos límites que están faltando en la pantalla para los correspondientes ejes de la ecuación. En este caso se los Ilamarán Xp0 y Yp0, para referirse a que son los puntos 0 de la pantalla tanto en x como en y. Asignando estos puntos al gráfico anterior se tendrán las siguientes coordenadas importantes alrededor de la pantalla, como se indica en la figura 4. 


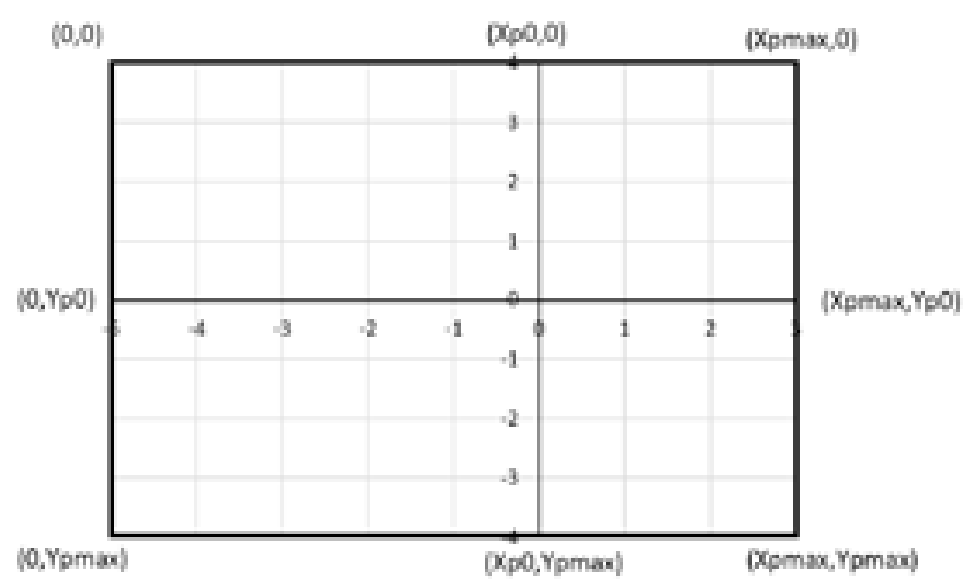

Figura 4. Asignación de puntos límites importantes de la Figura 3

Consecuentemente, tanto en el interior, como en el contorno del gráfico se visualizan etiquetas de todos los puntos importantes que se utilizarán en el proceso. Es importante recordar que aquellos puntos que hacen referencia a la pantalla tienen una " $p$ " en su designación y aquellos que harán referencia a la ecuación carecerán de dicha " $p$ ".

En el ejemplo que se está trabajando, por la designación tomada para la visualización, el punto Yp0 está justo en la mitad de la pantalla para el eje y, por lo que se podría sustituir a Yp0 porYpmax/2, pero a fin de dejar el ejemplo lo más general posible, no se hará esta sustitución.
4. Se trabaja con cada cuadrante y cada eje coordenado por separado. Con el fin de transformar los puntos de la ecuación en puntos de la pantalla se obtienen fórmulas a partir del cálculo de la distribución de la pendiente en cada cuadrante y cada eje coordenado. Estas ecuaciones permitirán transformar los valores de la ecuación que se desea graficar en puntos que pueden ser pixeles en el interior de la pantalla. Para facilidad de comprensión y para demostrar que el proceso es independiente en cada cuadrante, en lugar de ir analizando en orden de cuadrantes del plano cartesiano, se irá analizando en orden de dibujo desde la esquina 
superior izquierda a la esquina inferior derecha, es decir se analizará en el siguiente orden de cuadrantes: I, I, III, IV. Esto no significa que siempre se deba seguir este orden, operar en el orden de cuadrantes que se desee, incluyendo el que matemáticamente podría considerarse más adecuado I, II, III, IV; pero si se lo hiciera de este modo en el ejemplo que se plantea, la comprensión se podría ver afectada por no seguir un orden adecuado para el dibujo que se pretende realizar.

\section{a. Cuadrante II}

En este cuadrante, fijándose únicamente en la ecuación, los límites de la ecuación inician en 0 y terminan en un Xmax, y en y del mismo modo, desde 0 hasta Ymax. En el caso particular del ejemplo, $X \max =3$ y $Y \max =4$, pero para obtener fórmulas más generales, no se reemplazarán estos valores si no hasta el final de la explicación del procedimiento.

En este mismo cuadrante, ahora fijándose únicamente en la pantalla, se aprecia que se intenta dibujar la sección superior izquierda de la pantalla. Para esta sección los límites de la pantalla en $\mathrm{x}$ son de 0 a Xp0, y los de y son de 0 a Ypo. Ver Figura 5.

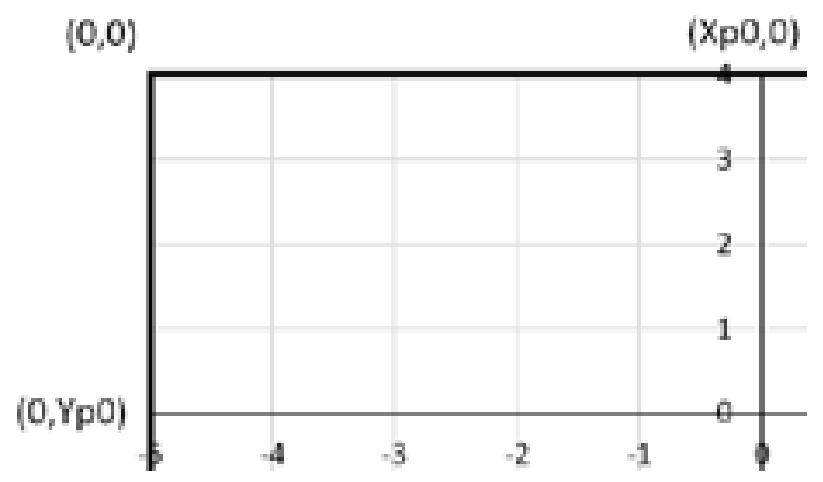

Figura 5. Cuadrante Il de la ecuación

Un punto $(x, y)$ cualquiera de la ecuación en este cuadrante debe ser transformado en un punto (xp,yp) de la pantalla que también se ubique 
dentro de los límites de $(0,0)$ hasta (Xp0,Yp0).

\section{Trabajando únicamente con el} eje $x$ :

En la ecuación la distribución es de Xmin a 0, en la pantalla la distribución es de 0 a XpO. Es decir, cuan- do en la ecuación se esté en el Xmin, en la pantalla se debería estar en 0 y cuando en la ecuación se llegue a 0 , en la pantalla debería llegarse a Xp0. Al graficar estos puntos en un eje coordenado que nada tiene que ver con el de la ecuación original, se tendría:

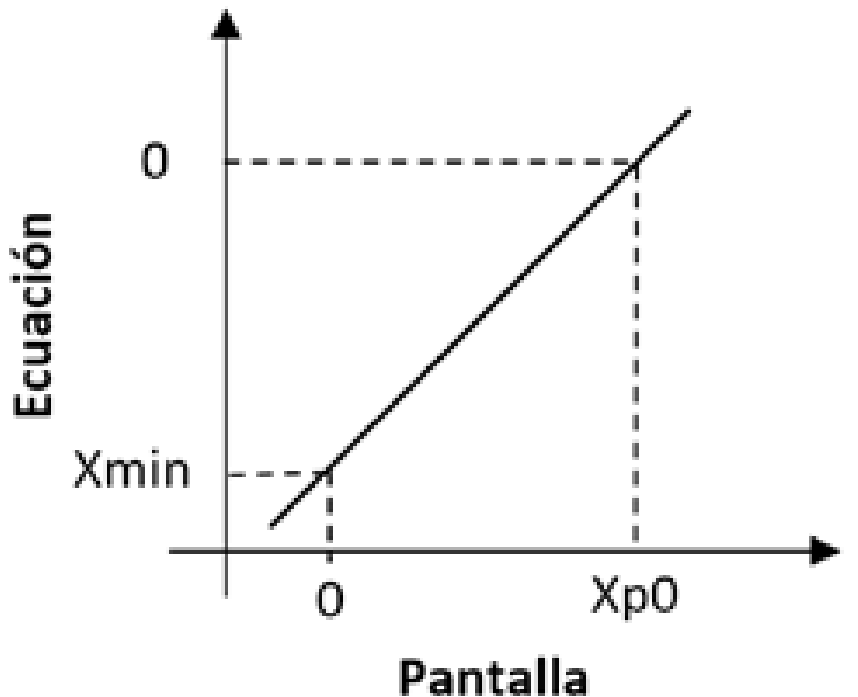

Figura 6. Distribución del eje x en la ecuación y la pantalla

Como se asume que debe existir un punto en la ecuación dentro de los límites indicados, que siga la misma distribución con respecto a la pantalla, se puede colocar un punto cualquiera de la ecuación hacia una correspondencia en la pantalla (Ver Figura 7). Ya que la pendiente del ángulo en el triángulo grande es la misma que la del triángulo pequeño, se puede igualar el cálculo de las pendientes y obtener la ecuación 
de transformación para puntos de para al eje x en el segundo cuadrante. la ecuación en puntos de la pantalla

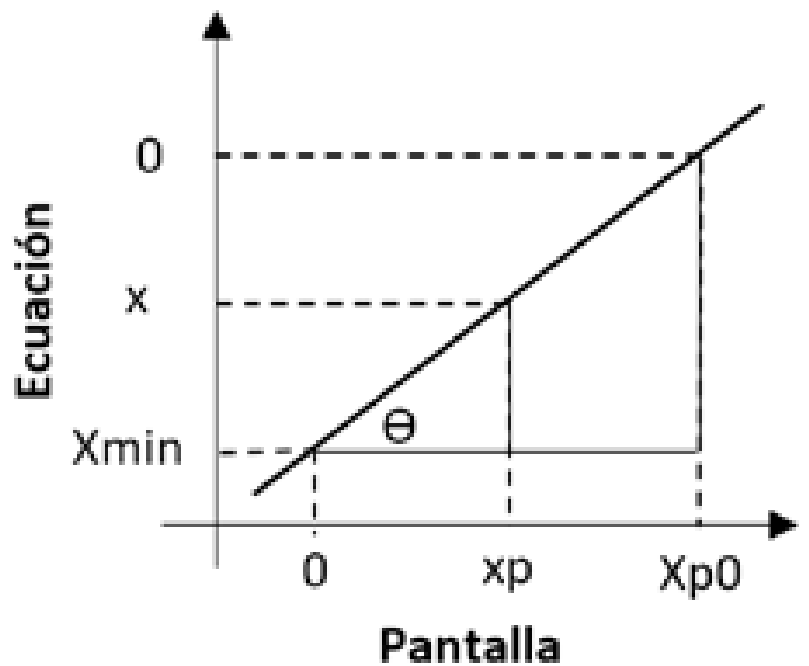

Figura 7. Pendiente para el eje x del cuadrante II

Por lo tanto, las pendientes serían:

$\tan (\theta)=\frac{\Delta y}{\Delta x}=\frac{0-X \min }{X p 0-0}=\frac{x-X \min }{x p-0}$

$Y$ al despejar el punto $x$ de la pantalla:

$$
x p=\frac{X p 0(x-X \min )}{0-X \min }
$$

Ahora, trabajando únicamente en el eje y:

Siguiendo el mismo proceso que se aplicó en el eje $x$, ahora para el eje y en el segundo cuadrante, los límites de la ecuación son de Ymax a 0 en correspondencia con los límites de la pantalla que van de 0 a YpO. Si se tiene en cuenta que ahora cuando en la ecuación es 0 , en la pantalla será Yp0, mientras que cuando en 
la ecuación sea Ymax, en la pantalla debe ser 0 , esto se debe a que a diferencia del eje $x$, en donde y la ecuación y los valores de la pantalla se incrementan directamente; en el eje y los valores cambian inversamen- te. Es decir, mientras en la ecuación aumenta de valor, en la pantalla disminuye, por lo tanto, el gráfico será inversamente proporcional, sin embargo, el cálculo de la tangente sigue obteniéndose del mismo modo.

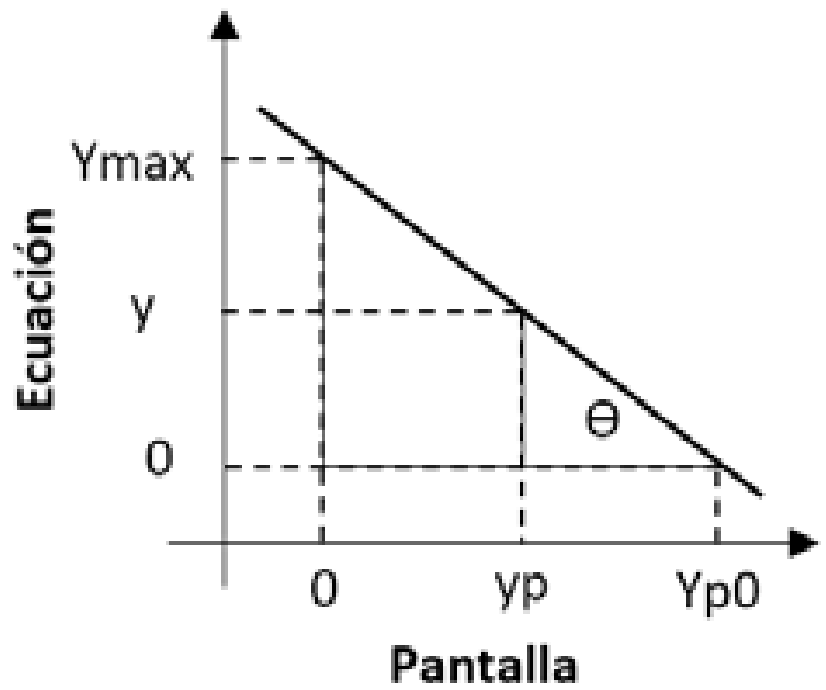

Figura 8. Pendiente para el eje y del cuadrante II

Al obtener las pendientes:

$$
\tan (\theta)=\frac{\Delta y}{\Delta x}=\frac{Y m a x-0}{Y p 0-0}=\frac{y-0}{Y p 0-y p}
$$

Y despejando el punto y de la pantalla:

$$
y p=\frac{Y p 0 * Y \max -Y p 0 * y}{Y \max }
$$

Al recapitular, la manera de transformar puntos desde la ecuación hacia la pantalla, siempre que se esté en el segundo cuadrante del plano cartesiano para la ecuación viene dado por las coordenadas: 


$$
(x p, y p)=\left(\frac{X p 0(x-X \min )}{0-X \min }, \frac{Y p 0 * Y \max -Y p 0 * y}{Y \max }\right)
$$

Ecuación 2: Ecuación de transformación final de coordenadas para el cuadrante II

\section{b. Cuadrante I}

El proceso es exactamente el mismo que se hizo para el cuadran- te II, pues al cambiar únicamente los límites a lo que corresponde en este cuadrante:

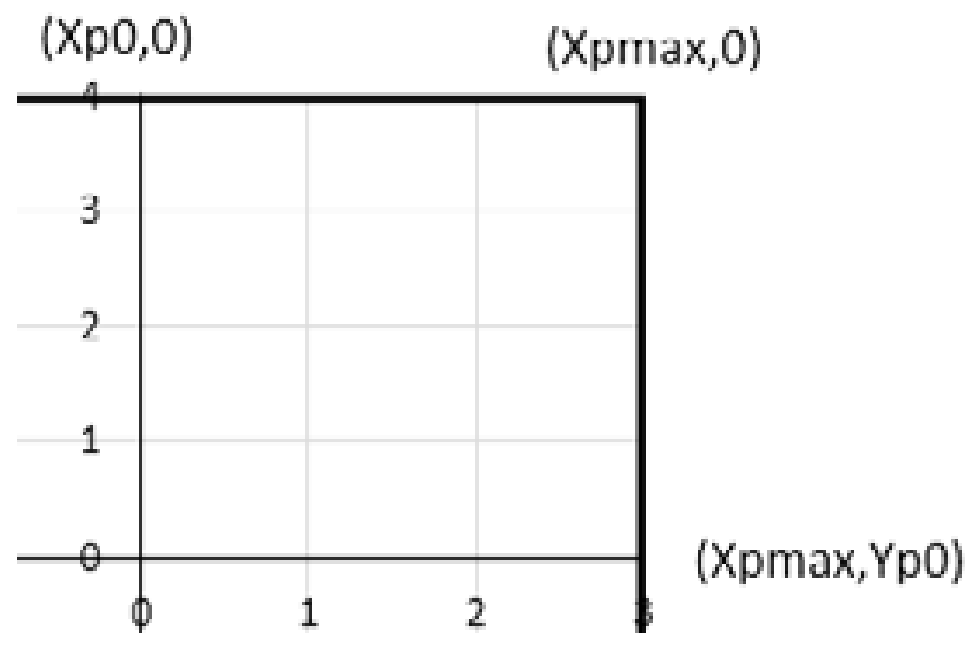

Figura 9. Cuadrante I de la ecuación 
Eje x: Se realiza el mismo análisis y se obtiene la siguiente gráfica:

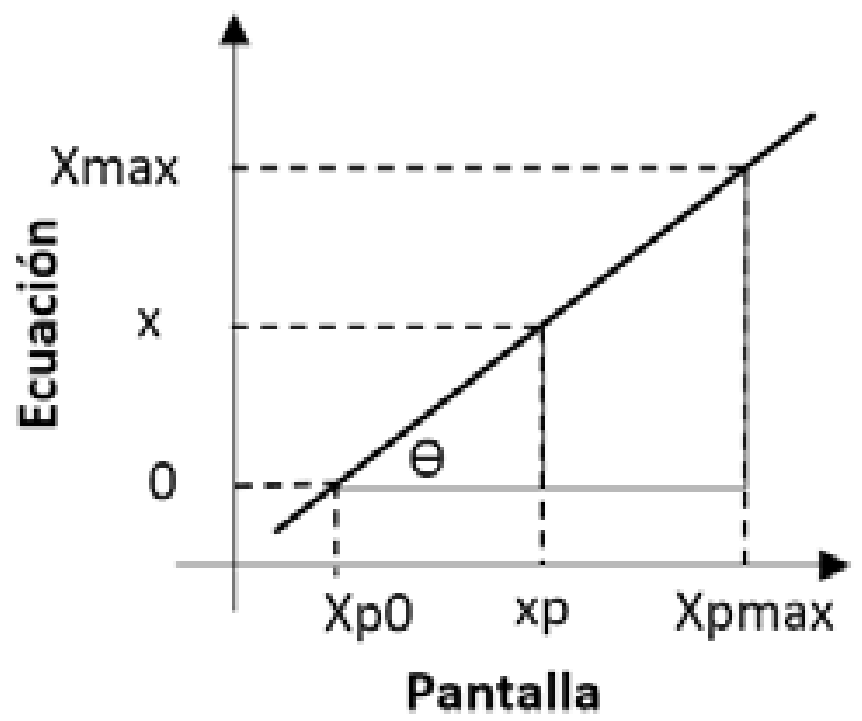

Figura 10. Pendiente para el eje x del cuadrante I

Pendientes:

$\tan (\theta)=\frac{\Delta y}{\Delta x}=\frac{X \max -0}{X p \max -X p 0}=\frac{x-0}{x p-X p 0}$

Despejando el punto $x$ de la pantalla:

$$
x p=\frac{x(X \max -X p 0)+X \max * X p 0}{X \max }
$$


Eje Y: Se realiza el mismo aná-

lisis y se obtiene la siguiente gráfica:

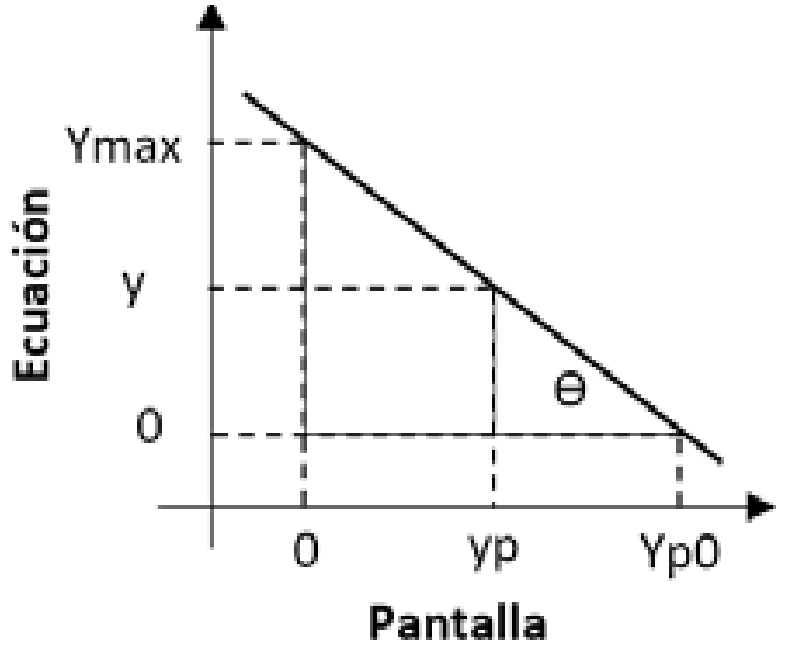

Figura 11. Pendiente para el eje y del cuadrante I

Pendientes:

$\tan (\theta)=\frac{\Delta y}{\Delta x}=\frac{Y \max -0}{Y p 0-0}=\frac{y-0}{Y p 0-y p}$

Despejando el punto y de la
Así que las coordenadas de la pantalla (xp, yp) para puntos $x, y$ de la ecuación en el primer cuadrante serían:

pantalla:

$$
\begin{aligned}
y p & =\frac{Y \max * Y p 0-y * Y p 0}{Y \max } \\
(x p, y p) & =\left(\frac{x(X \max -X p 0)+X \max * X p 0}{X \max }, \frac{Y \max * Y p 0-y * Y p 0}{Y \max }\right)
\end{aligned}
$$

Ecuación 3: Ecuación de transformación final de coordenadas para el cuadrante I 


\section{c. Cuadrante III}

Para este momento el mecanismo debe haber sido comprendido, por lo que solamente se colocarán las gráficas y ecuaciones correspondientes, tanto en el tercero como en el cuarto cuadrantes.

Cuadrante en análisis:

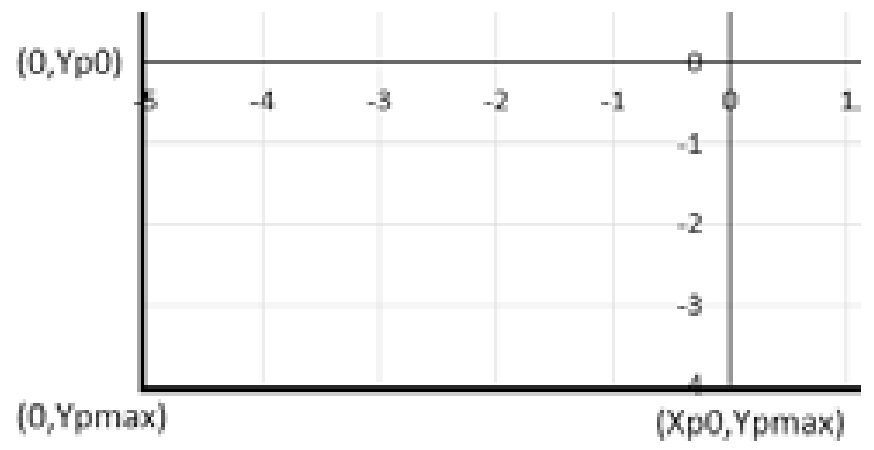

Figura 12. Cuadrante III de la ecuación

\section{En x:}

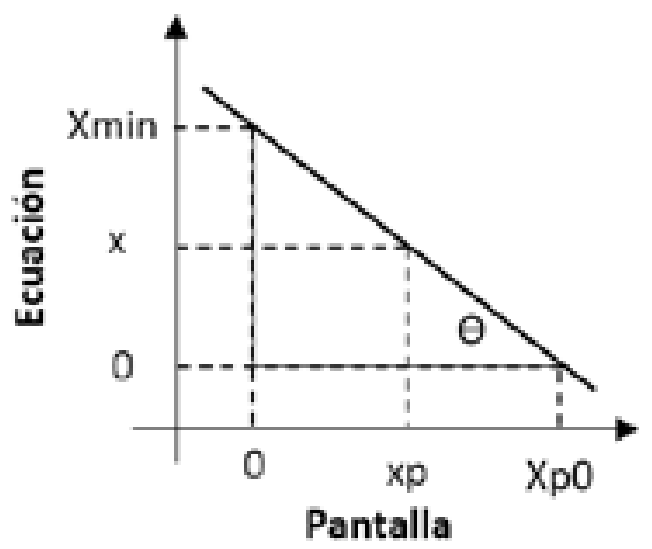

Figura 13: Pendiente para el eje x del cuadrante III 
Calculando y despejando:

$$
x p=\frac{X \min * X p 0-x * X p 0}{X \min }
$$

\section{En y:}

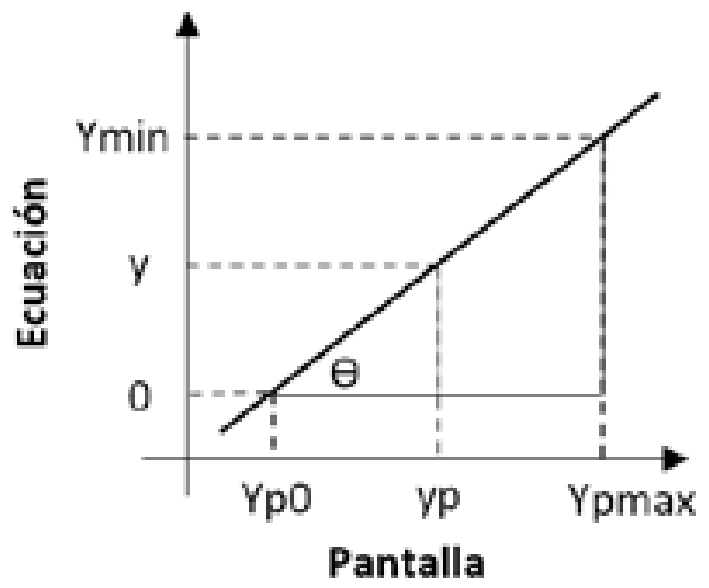

Figura 14. Pendiente para el eje y del cuadrante III

Calculando y despejando:

$$
y p=\frac{y(Y p \max -Y p 0)+Y p 0 Y \min }{Y \min }
$$

Por lo tanto, Cordenadas:

$$
(x p, y p)=\left(\frac{X \min * X p 0-x * X p 0}{X \min }, \frac{y(Y p \max -Y p 0)+Y p 0 Y \min }{Y \min }\right)
$$

Ecuación 4: Ecuación de transformación final de coordenadas para el cuadrante III 


\section{d. Cuadrante IV}

Cuadrante en análisis:

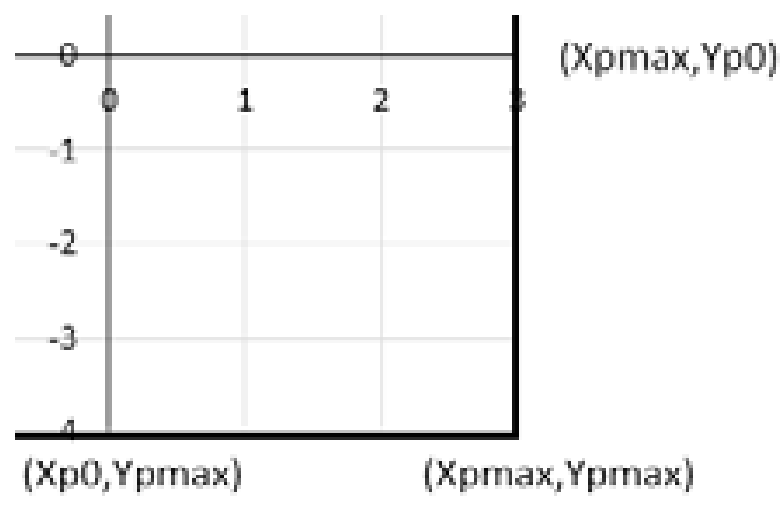

Figura 15. Cuadrante IV de la ecuación

\section{En x:}

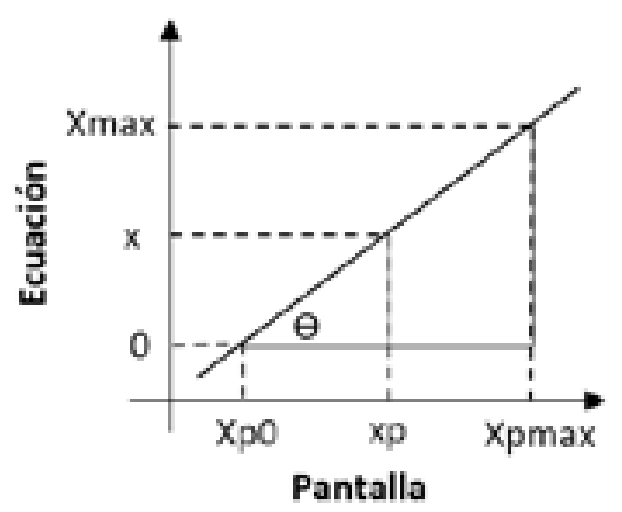

Figura 16. Pendiente para el eje x del cuadrante IV 
Calculando y despejando:

$x p=\frac{x(X p \max -X p 0)+X \max * X p 0}{X \max }$

En y:

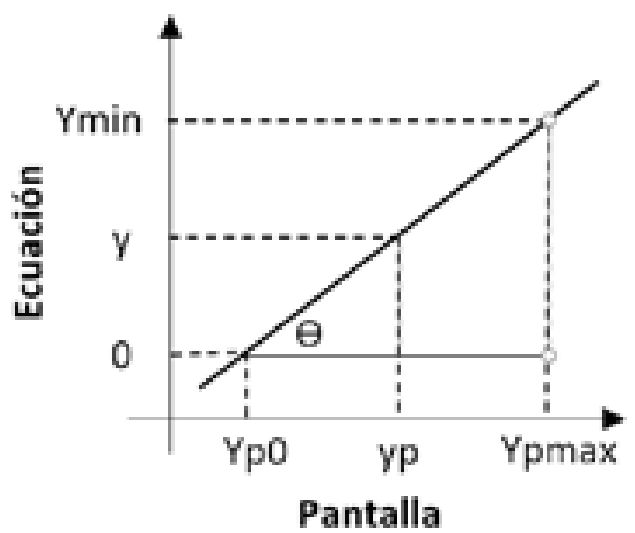

Figura 17. Pendiente para el eje y del cuadrante IV

Calculando y despejando:

$y p=\frac{y(Y p \max -Y p 0)}{Y \min }+Y p 0$

Por lo tanto, Coordenadas:

$$
(x p, y p)=\left(\frac{x(X p \max -X p 0)+X \max * X p 0}{X \max }, \frac{y(Y p \max -Y p 0)}{Y \min }+Y p 0\right)
$$

Ecuación 5: Ecuación de transformación final de coordenadas para el cuadrante IV

5. Se hace una impresión por pixeles para la ecuación desde $x=$ Xmin hasta $\mathrm{x}=$ Xmax y se aplica la fórmula que corresponda, según el cuadran- 
te en el cual esté operándose x. La fórmula que se utilice dará un punto coordenado $(x, y)$ que podrá dibujarse en la pantalla en el lugar que corresponda de la ecuación gracias a la transformación de escalas.

Al retomar el ejemplo inicial y al aplicar las ecuaciones encontradas en un punto aleatorio cualquiera de la Ecuación 1, en un cuadrante cualquiera, dentro de los límites establecidos, se transformará el punto aleatorio de la ecuación al correspondiente punto de la pantalla.

Retomando los valores calculados necesarios, descritos en el paso 3 del procedimiento, se obtiene:

Ecuación: $X \min =-5, X \max =3$, $Y \min =-4, Y \max =4$
Pantalla: Suponiendo que se trata de un cuadro de dibujo de $800 \times 600$, entonces $X p m a x=800$, Ypmax $=600$. Calcular Xp0 y Yp0 no es difícil, ya que se sabe que debe ser la misma proporción respecto de la ecuación, por lo tanto, se debe hacer unos sencillos cálculos, $\mathrm{Xp0}=500$ y $Y p 0=300$.

El punto que se ha decidido tomar aleatoriamente para la ecuación 1 es $x=2$, por lo tanto: Si $x=-2$, reemplazando en esa ecuación, se obtiene que $y=2$. El punto $(-2,2)$ se sabe que se ubica en el segundo cuadrante del plano cartesiano, por lo que para transformar en un punto en la pantalla se debe usar la ecuación 2 de las ecuaciones de transformación obtenidas en el literal a del paso 4 del procedimiento:

$$
(x p, y p)=\left(\frac{X p 0(x-X \min )}{0-X \min }, \frac{Y p 0 * Y \max -Y p 0 * y}{Y \max }\right)
$$

Reemplazando:

$$
(x p, y p)=\left(\frac{500(-2-(-5))}{0-(-5)}, \frac{500 * 4-500 * 2}{4}\right)=(300,250)
$$

Es decir, el punto $(-2,2)$ de la ecuación corresponde al punto $(300,250)$ en la pantalla, según los límites definidos, tanto para la ecuación como para la gráfica.

Finalmente, cualquier punto $(x, y)$ de la ecuación entre Xmin y Xmax puede ser transformado en un correspondiente punto de la pantalla aplicando la ecuación que corresponda dentro de las 4 encontradas en el paso 4 del procedimiento. Si se desea utilizar otros límites diferentes a los determinados en los pun- 
tos 1, 2 y 3 del procedimiento, se debería volver a realizar los cálculos de pendientes necesarios para encontrar las nuevas ecuaciones de sustitución.

Se ha elaborado un software en plo propuesto. Este software se puede encontrar en el siguiente repositorio: https://goo.gl/YiNZAS

El código fuente es el siguiente:

C\# que aplica este método para el ejem-

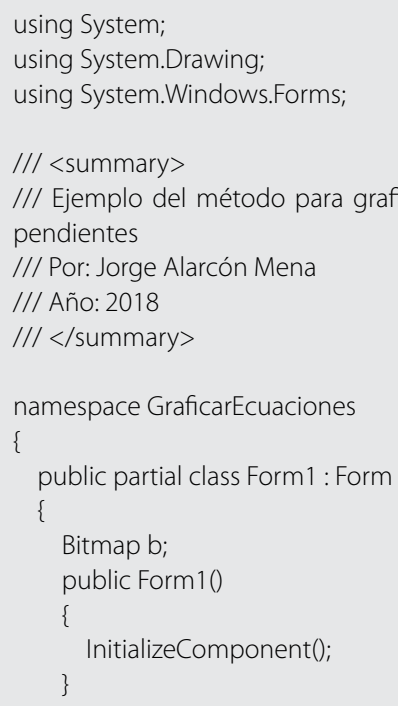


podría hacer un recorrido con mayor distancia si en lugar de puntos se dibujaran líneas.

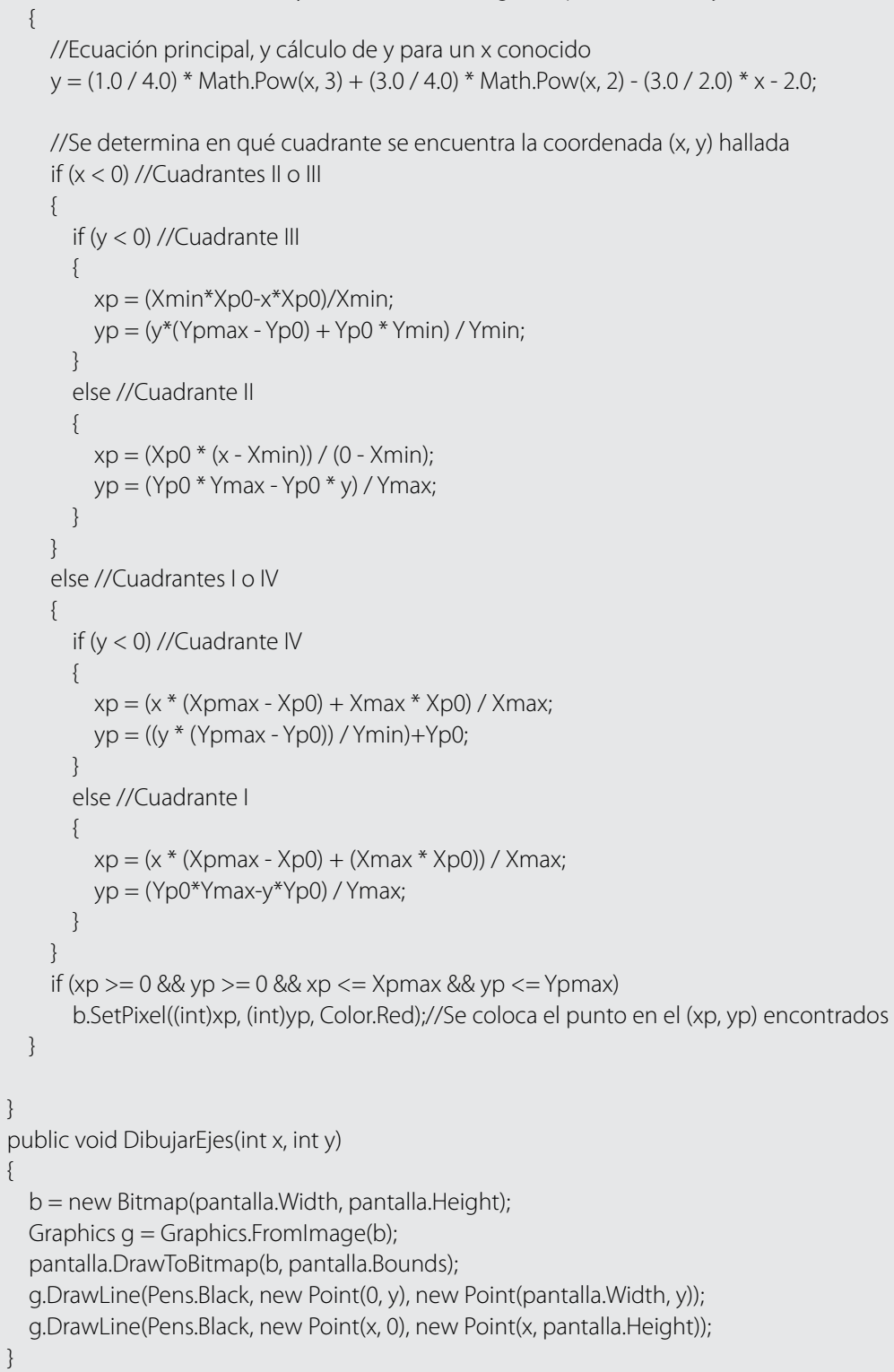


private void pantalla_Paint(object sender, PaintEventArgs e)

\{

if ( $b !=$ null)

pantalla.Image $=($ Image $)$ b.Clone();

\}

\}

\}

La pantalla resultante de la ejecu-

ción es la siguiente:

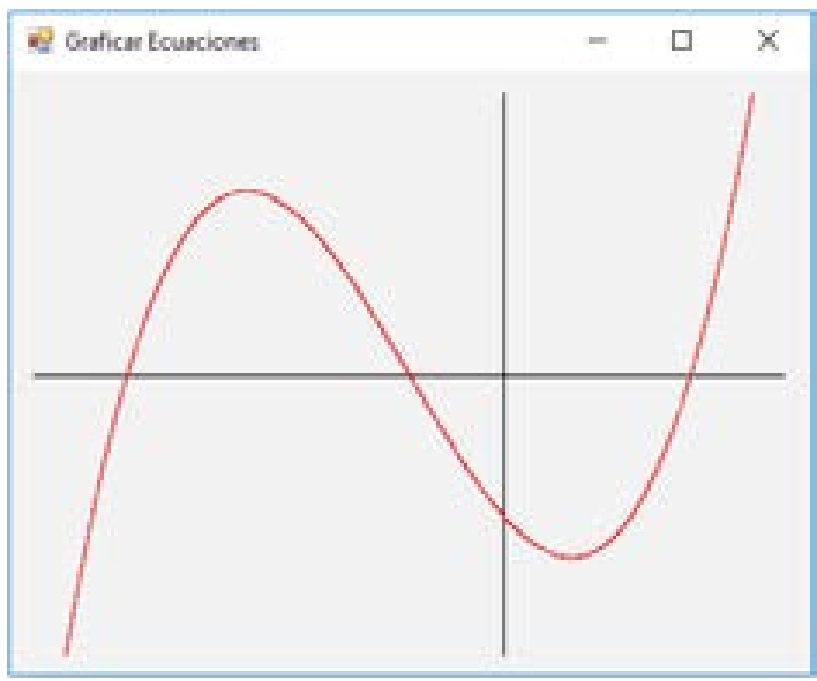

Figura 18. Ejecución del software de aplicación del método 


\section{CONCLUSIONES}

Hay una diversidad de maneras para transformar puntos de un sistema de coordenadas conocido a un objeto gráfico que se reflejará en la pantalla de un computador.

Esta propuesta está centrada en la graficación de ecuaciones utilizando la distribución que la ecuación tiene sobre los ejes $x$ e y en cada uno de los cuadrantes en los que se desee dibujar.

Es posible que las fórmulas encontradas en este proceso se utilicen universalmente cuando se desee graficar ecuaciones en los 4 cuadrantes, pero más importante que ello es el modo mediante el cual se obtuvieron dichas fórmulas, ello permite estandarizar el proceso para cualquier número de cuadrantes en dos dimensiones, por ejemplo, si la ecuación se desearía graficar solamente en el primer cuadrante.

También se puede concluir que las condiciones iniciales no son una camisa de fuerza una vez que se entiende cómo trabaja el método propuesto. Por ejemplo, una de esas condiciones era que la ecuación sea continua y exista para todo el rango en el que se va a dibujar. Esta condición podría llegarse a no ser tomada en cuenta, si no se aplican las fórmulas encontradas para el rango en el cual la ecuación no existe, pero se debería encontrar un proceso que deje el espacio suficiente que refleje la no existencia de la ecuación en el rango correspondiente al momento de graficar. 


\section{BIBLIOGRAFÍA}

Brice-Arnaud, G. (2016) ASP.NET en C\#Con Rex, B. (2015), Principles and Practice of Visual Studio 2015, Barcelona: ENI. Núñez, M. (2002), Física 2, México: Limusa. Structural Equation Modeling, New York: The Guilford Press.

Torres, J. (2014), Matrices y Sistemas de Ecuaciones Lineales, Medellín: Universidad de Medellín.

vias al Cálculo, Medellín: Universidad De Medellín. 